\title{
How to cope with different medical cultures in Europe
}

\author{
London, September 24th-25th, 1992
}

\author{
Robert Randling \\ Trumpington, Cambridge, UK \\ (Accepted 15 September 1992)
}

\begin{abstract}
Are doctors better in one part of Europe than another? Or do they merely differ in a manner perhaps reflecting real or perceived differences in the needs of the people whom they treat? The questions were among those which came to mind repeatedly at the novel and instructive conference which IBC Technical Services organized in London in September. Neither of the questions was entirely answered, but a great deal of fodder was provided for those who want to resolve them. The demand for answers is growing; variations in medical culture which seemed merely curious three decades ago - Lynn Payer's book is the classic repository of anecdotes on the subject, but it is really no more than that - are becoming important as the European Community struggles towards its ideal of the truly free movement of goods and services across frontiers. If the differences which clearly exist - not merely in doctors but also in services, health concepts, experiments in man and indeed every aspect of health care and science - can be sufficiently documented as significant, they will either need to be justified or they will have to be eliminated wherever they stand in the way of proper care.

Some of the firmer evidence on the matter might be expected to come from the market researchers who are of necessity rather more practically minded than certain sociologists. Colin Maitland proved to have distilled from this market research - largely undertaken for the pharmaceutical industry - a portrait of the more traditional differences between the doctors of the nations. French doctors he concluded, were thinkers, spending a great deal of time listening to their patients, preferring gentle therapy to drastic; reflecting the beliefs of their patients, however, they devoted much attention to the liver and the digestive system, and ended up as likely as not prescribing suppositories. Spanish doctors were traditionally less well paid, unhappy with their status, yet singularly reluctant to refer their patient to the specialists. As to the British doctor, he appeared to work less hard than the German, make greater use of his locum, seek for mechanical solutions to problems
\end{abstract}


(devoting a great deal of attention to the bowels) and dispatch his patient from the consulting room after a mere six minutes... Whatever truth there may be behind such portraits, Mr Maitland was the first to suggest that they are becoming less lifelike with time as the physician across Europe is subject to similar pressures from patients, advertisers and the tightening economy. Health care in Germany currently runs at $13.1 \%$ of G.N.P., as compared with $6.7 \%$ in Britain and $5.2 \%$ in Spain; would the grundlichkeit of the German physician survive a sixty percent reduction in health funding to U.K. levels? Mr Maitland necessarily left his audience wondering uncomfortably whether the trend to sameness in doctors is not a trend towards the lowest possible level of care in medicine.

One might expect evidence of injury in health care to provide some evidence of the state of the art. Where drugs were concerned, Ralph Edwards and Marie Lindquist (whose papers will appear elsewhere in this Journal) urged caution; there are indeed differences in the rates at which reports of adverse effects appear from country to country, but that may reflect divergences in method rather than in medicine; one is reminded of the fact that in those parts of the world where medicine is at its lowest ebb there seem to be no reports of adverse reactions at all.

At times, the meeting heard the rebuke that, as it proceeded to hear long accounts of multi-national ventures in clinical research and drug marketing, it was deviating from its expressed goal. Yet notably it is precisely in these fields that some of the greatest difficulties occur in dealing with Europeans as if they were a single race. The Northern European Physician, asserted Mr Maitland, will react to forms of persuasion which are factual, documented, calculated to appeal to the left side of the brain; move south, and emotional arguments, suggestion, testimonials and personal persuasion are the instruments in demand. One could distil similar distinctions in culture and tradition from the manner in which attempts to protect the subject of clinical experimentation have been brought into effect. It is entirely true, as Holland's Wim Rosinga demonstrated for the meeting, that most countries have chosen ethical committees, informed consent and compulsory insurance as the three tools needed to protect the experimental subject; all the same, those tools have been applied in different ways, with legislation, regulation, local initiative and voluntary effect variously playing the essential role, depending on the manner in which a nation has chosen to organize itself. On one side of a frontier self-regulation is simply a great deal more likely to emerge (and to be effective) than on another. One would add that Dr Rosinga succeeded almost unintentionally in protraying another facet of national differences as regards the effectiveness of national edicts and of the administrative organs which are supposed to bring themselves into effect; there are still a number of places in Europe where social laws seem to be made merely in order to play to the gallery, subsequently to languish unimplemented in the statute books.

Christopher Hodges drove a similar lesson home as regards the manner in which medical accidents are handled; though the meeting's emphasis on the countries of the Community led him to say a great deal about the laborious emergence of the Product Liability Directive, he was the first to admit its defects. The manner in which the Directive defines a product defect and the harshness of 
its ten-year period of limitation betray the fact that its authors had motorcycles and electric irons in mind rather than medical treatments and drugs. All in all, $\mathrm{Mr}$ Hodges might have been a little kindlier to the compensation schemes which have come into being in several non-community countries, notably in Sweden, and which would appear to operate more quietly, quickly and effectively than the bringing of cases under the Product Liability Directive is likely to do; alas, it may be a long time before we know, for despite all the effort put into the Directive no-one (to $\mathrm{Mr}$ Hodge's knowledge) appears so far to have made any use of it.

The final measure of European homogeneity in health matters will probably only be delivered if and when patients and doctors begin to move across the continent as freely as they are now in theory entitled to do. Patients most certainly do move a great deal, but primarily as true tourists; when the ague takes them they may well be found to hurry to a medical compatriot on the Costa Brava or to return home. As to doctors, the small print in the regulation still stands very largely in the way of their migration, with subtle demands that the doctor on the move first prove his competence wherever he may go; if that is not sufficient impediment, there is the language barrier; and beyond that there is still the barrier of culture. If the figures are to be credited, there is still rather more medical movement around the North Sea and across the Mediterranean than between the northern and southern poles of the Community itself.

The meeting therefore did not provide a firm answer to the vexed and sensitive question of medical quality, though there seemed to be consensus among participants in the debate that the manner in which a doctor practices is moulded largely by his circumstances. The physicians of one nation may, on emergence from medical school be much better versed in theory and less attuned to practice than those of the next; but they will thereafter spend a period forgetting the one and learning the other (or vice versa) until they become relatively alike. So it may be, but the anecdotes will long remain, as will the need for study. As the Chairman (curiously from the World Bank) remarked in closing: if we are ever finished with the conundrum of medical diversity in the West it will be just in time to start looking at the same matters further; shall we next time need to meet in Minsk? 